\title{
Editorial
}

\section{Meeting challenges in delivery of patient care: a reflection on the involvement of ERS in CPD of respiratory physicians}

At the end of his mandate, Gernot Rohde (European Respiratory Society (ERS) Education Council Chair 2014-2017) and the ERS Education Council had the opportunity to reflect on the goals achieved over 3 years and the challenges in implementing continuing professional development (CPD) programmes for our respiratory specialists. The third ERS Education Research Seminar took place in Frankfurt, Germany, in June 2017 with >30 international experts in medical education and respiratory medicine. $>1$ year later, we can now reflect on the ERS Education Seminar, the achievements of the Education Council over 3 years, and the continuing path to better understand the education needs of practicing respiratory medicine specialists. This article reflects on the core principles of medical education and offers a reflection on CPD for respiratory professionals within ERS Education.

\section{An overview of CPD}

Most European countries have recognised the need to encourage and, to some degree, "regulate" CPD for physicians in practice that is used for recertification. Within the scope of lifelong learning, CPD requires that healthcare professionals need to stay up to date with current developments in knowledge and skills to remain fit to practice [1]. The rationale of introducing CPD systems is to support physicians to maintain their proficiency and safeguard patients by ensuring that healthcare professionals are competent to provide safe and effective care to their patients throughout their career. However, critique of existing systems of CPD is mounting within the literature [2, 3], based around CPD's ineffectiveness in supporting the physician's personal performance, or having any impact on patient outcomes and so improving patient outcomes. The study by SEHLBACH et al. [4], supported by the ERS, explored respiratory specialists' acceptance and perception of effectiveness of different national systems [5]. It shows that without quality control, collection of CPD credits can turn into a box-ticking exercise, reducing physicians' trust in the system. Engaging physicians in designing CPD systems can help to increase engagement and commitment, ultimately improving learning culture and patient care. A prior study investigated the disparities, purpose and perceived need for lifelong learning for medical specialists. The authors shed light on current national recertification systems and provided further rationale for reform [5]. Mobility of patients and physicians across European borders not only calls for standardisation of training and qualifications on entry into practice [6] but also requires consideration of standards for CPD [7]. Using CPD for practice improvement is gaining increased attention from scholars and policy makers alike [3]. International medical societies have the unique opportunity to set best practice standards and provide a framework for medical specialists to follow. International societies such as the ERS can, however, only recommend best practice standards as regulatory powers remain with national bodies [5]. Despite this, international societies could require their members to prove that

@ERSpublications

A reflection on @EuroRespSoc education, a learning pathway for the future: better practice, better physicians, better patients http://bit.ly/2XHmr3j

Cite as: Mitchell S, Sehlbach C, Driessen E, et al. Meeting challenges in delivery of patient care: a reflection on the involvement of ERS in CPD of respiratory physicians. Breathe 2019; 15: e97-e103.

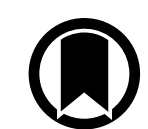

CrossMark 
they are reflective learners and engage in continuing professional development. In turn, physicians may be able to use internationally collected credits for their national CPD programme.

\section{Pillars of education: supporting a structure for continuing professional development}

ERS Education is structured with four core pillars:

- curriculum development

- educational programmes

- electronic learning (e-learning)

- assessment

with each pillar playing a key role in the design, planning and delivery of practice-based education and certification of trained specialists to support and improve professional performance. The curriculum builds the foundation of educational programmes, identifying the learning outcomes (knowledge, skills and attitudes), core competencies based on physician competency frameworks such as CanMEDs [8] and a level of assessment for certified programmes, as well as identifying teaching and learning opportunities. The programmes concentrate on the delivery of teaching and learning activities. Currently, the content is focussed around the eight major respiratory diseases, and 11 key methods in basic, translational, clinical and epidemiological research and clinical practice (table 1). The programmes cover knowledge and skills acquisition, utilising the range of teaching and learning methods, in various settings from ERS International Congress sessions to bedside clinical observation. The programme pillars also incorporate faculty development, supporting the development of teachers, trainers and assessors for all ERS educational and scientific activities. E-learning additionally falls within teaching and learning by providing faculty and participants with online educational tools. Simulation, electronic portfolios, online quizzes, video procedures, gaming and many other tools have recently gained considerable attention within medical education. Assessments drive learning and ensure that those taking part in a programme are competent to practice. The ERS has specifically identified the need to focus on different types of assessment methods by professional group (figure 1), shifting from summative assessment for in-training and post-training levels to formative assessment with feedback for CPD.

\section{Different needs for different audiences}

If we consider the diverse learning needs of individual trained respiratory specialists within the ERS, to provide a standardised, systematic or mandatory approach will probably fail to improve CPD. The process, structure and rationale for CPD for recertification is rooted within national systems of governance and policy. Hence, the development of a concrete international system for recertification in respiratory medicine may not be acceptable, feasible or applicable to our members, and therefore should not be the primary focus of the ERS. However, by recognising inconsistencies and shortcomings in current national systems, the ERS has a real and significant role to play in establishing a framework for best practice, and providing educational programmes to support and improve performance for trained specialists and delivery of care to patients.

The ERS embraces different professional groups across the spectrum of respiratory medicine. It is no surprise therefore that the challenges faced by respiratory clinicians may differ from those faced by thoracic surgeons or allied health practitioners, or those in training, working in private practice or an academic hospital. The first challenge is

Table 1 European Respiratory Society major respiratory diseases, key methods and professional groups

\begin{tabular}{lll}
\hline Major respiratory diseases & $\begin{array}{l}\text { Methods in basic, translational, clinical and } \\
\text { epidemiological research and clinical practice }\end{array}$ & Professional groups \\
\hline Respiratory critical care & General respiratory patient care & Adult pulmonologists/clinicians \\
Sleep and breathing disorders & Respiratory intensive care & Scientists (basic and translational) \\
Airway diseases & Pulmonary function testing (including gas exchange) & Clinical researchers \\
Paediatric lung diseases & Endoscopy and interventional pulmonology & Paediatricians \\
Respiratory infections & Imaging & Thoracic surgeons \\
Thoracic oncology & Surgery & Thoracic oncologists \\
Interstitial lung diseases & Transplantation & Radiologists \\
Pulmonary vascular diseases & Epidemiology & Respiratory critical care physicians \\
& Physiology & Allied healthcare professionals \\
& Cell and molecular biology & General practitioners \\
& Public health & Physiologists
\end{tabular}




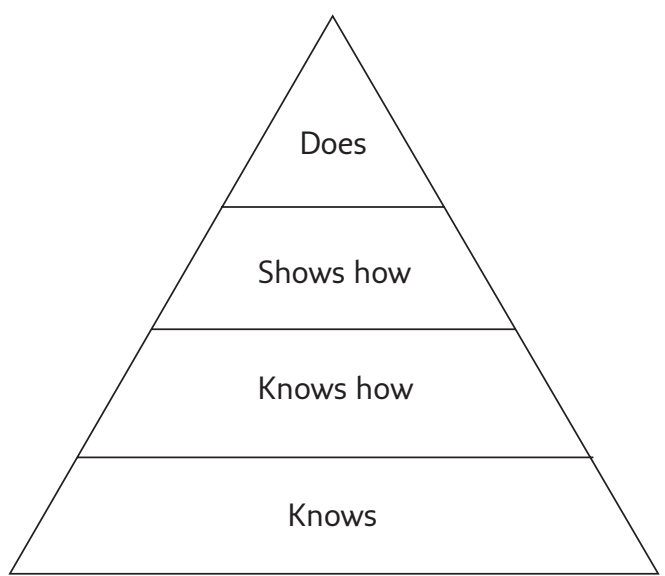

Figure 1 Miller's pyramid. Reproduced and modified from [9] with permission from the publisher.

to better understand the professionals' learning needs to ensure that they may be addressed in a feasible, structured and systematic approach. This is an ambitious task. As a first step, the different groups have been briefly summarised in figure 2 . In order to better understand the perceived need, the ERS has the opportunity to conduct a thorough investigation. Such an investigative study should involve a mix of methods including questionnaires, focus groups and one-to-one interviews across a cross section of members. The existing activities per group are discussed below.

"Trainees (in-training)" in respiratory medicine form not only the basis of the basic level of competence we expect of our newly trained respiratory specialists entering practice but also outlines the framework for what is expected for trained respiratory specialists to keep up to date in the field. The organisation and delivery of some ERS activities specifically target this group of members (e.g. curriculum for trainees 2005 and the seated HERMES knowledge-based multiplechoice questionnaire) [10].

The level of training required for "trained specialists in general adult respiratory medicine" is for trained specialists within ERS that deliver care to patients across the full spectrum of respiratory diseases and disorders. It will also include, to some degree, specialists in core topic areas who may have to deal with general respiratory patients referred to the respiratory unit for diagnosis and referral or management on call or during general outpatient clinics or supervision activities on the ward.

"Trained specialists and subspecialists in core topic areas" account for most ERS members and, to some degree, should reflect the structure of ERS assemblies. From research within ERS across the various topic areas, we know that training and certification at this level is very often "on the job" and dependent on the quality of training offered by the local trainer or training centre [11-14]. ERS has sought to prescribe standards for training in this core area, again for two groups.

- Trainees entering the "subspecialty" (delivery of competent and safe patient care)

- Trained subspecialists (delivery of excellent care to respiratory patients)

However, trained specialists in core topic areas also may have to perform on-call duties in a busy respiratory department for which the whole spectrum of respiratory medicine needs to be mastered in parallel.
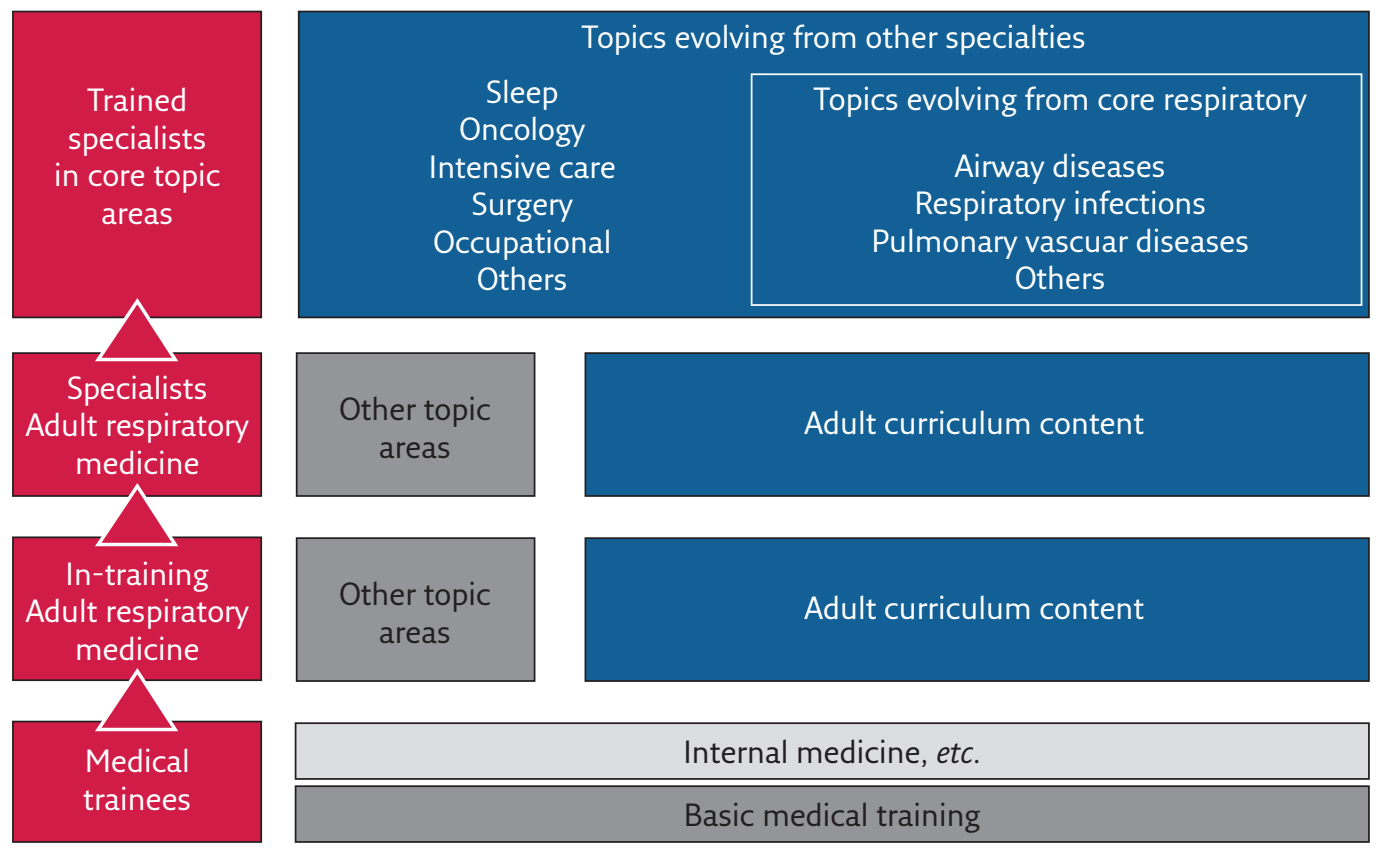

Figure 2 Professional groups across the spectrum of respiratory medicine. 
Key outcomes: 2017 Education research seminar

Educational research seminars are an opportunity to step out of the day-to-day running of activities to discuss the fundamental strategic direction for ERS Education. Building on from the two previous seminars in 2011 and 2014, key opinion leaders in respiratory medicine together with medical education experts convened in Frankfurt, Germany, in June 2017, to discuss the unique ERS CPD environment, challenges and future directions.

\section{The future of CPD for ERS: what we have learned during the seminar}

Topic identification and curriculum

Since the development of the first ERS curriculum on adult respiratory medicine, aimed at physicians in training [15], ERS has developed a significant number of curricula for respiratory specialist groups, which have been used internationally as a framework to guide training hospitals and national curriculum development. In addition, curricula have been developed for the complete implementation of two competency-based training programmes (endobronchial ultrasound and spirometry) covering clinical techniques [16]

With most professionals that follow ERS CPD activities being practicing clinicians, it was felt that there is a significant need to address the professional development needs of this group.

Key questions arose, such as:

- What knowledge and skills are needed to keep a general respiratory medicine specialist up to date?

- How can the training programme design be flexible enough for the different needs for respiratory clinicians?

- How can a practicing respiratory professional assess the gap in their knowledge, skills or attitudes; i.e. how does someone know what they do not know?

It was discussed that ERS Education could work together with ERS Assemblies to identify learning needs and methods for trained specialist to maintain their performance.

Programmes and e-learning

Programmes concentrate on delivering high-quality teaching and learning activities, and educational content. Over the past 10 years, ERS has been making large strides in building on its traditional lecture-based offerings to include a wider variety of blended teaching methods. During the Educational Research Seminar, the group looked at how to deliver activities that will meet the needs of the core target audience and ERS members and have a measurable impact on improved patient care, and how to prioritise topics and future developments. The participants discussed the greater role that online content was playing in professional development. It was agreed that the knowledgerelated learning objectives lend themselves well to online and individual activities. Participants can access these at their own time schedule and pace, allowing very flexible usage. However, using the potential of online educational content also comes with several challenges. Motivation and persistency of distance users is known to be challenged in online learning activities [17]. Furthermore, professionals do most of their learning in the workplace, and transfer of online acquired knowledge and skills to this workplace should not be taken for granted. Online teaching and learning activities should therefore be integrated with offline activities.

Use of portfolios was discussed as a potentially useful tool for reflection, monitoring and documenting progress within CPD, and validating professional development. Portfolios can contain evidence of how learners fulfil tasks and how their competence is progressing [18]. Even though they offer possibilities to stimulate professional development and performance at the "does" level, and could help ERS to cross borders between countries, training and career stages, caution was strongly advised. The importance of supervision, critical reflection, and support of planning and monitoring as well as the risk of creating bureaucratic overload were addressed. Mentoring, interviews and feedback from peers, colleagues and patients should be organised, and agreement and clarity about ownership, goals, competences, criteria and content should be made.

In the end, the discussion came down to the value assigned to these teaching and learning activities by professionals, local institutions and the ERS community.

\section{Assessments}

Mere exposure to an activity (e.g. performance of a certain number of procedures) or a doctor's self-perception of superior performance do not guarantee optimal practice; assessment is the most recognised way to ensure competency. Furthermore, assessment is a great motivator, drives learning and has been shown to increase retention [19]. However, formal certification and re-certification are performed on a national level, which is important to acknowledge when considering assessments in CPD by the ERS. The type of assessment must fit the intended purpose and target audience. Theoretical knowledge (lowest levels of Miller's pyramid) (figure 2) can be assessed by written tests or electronic testing, whereas practical skills must be assessed either in a simulation-based or work-based environment (the top two levels of Miller's pyramid). Dedicated 
assessment tools based on global rating scales, not checklists, are best suited to capture the finer nuances of experienced doctors' performance and can be used for both formative and summative assessment and to explore the efficacy of new training programmes [20].

Integrating several purpose-built assessments with solid evidence of validity can improve the quality of ERS' CPD programmes, and thereby enhance their credibility and value for the doctors, the hospitals and the patients [16]. One shortfall of this approach is the assessment of nonmedical/technical skills. During the seminar, it was discussed that the traditionally softer skills, such as communication, are being introduced as fundamental parts of modern curricula for medical schools and postgraduate training programmes in Europe. Experience was shared from the UK, where one of the cornerstones of reform of assessment for UK undergraduate and postgraduate medical training is the acceptance that a student/trainee will be assessed both by examinations and in the workplace. The programme of assessment methods must be integral to the curriculum. Taken together, this ensures assessment of each domain of Good Medical Practice, the UK General Medical Council's requirement and guidance for the profession. It requires us to design and provide assessment tools for looking at actual clinical practice, for assessing real consultations with real patients who are consulting about their own problems, in the language spoken by the patient. Whilst ERS can help set the standards for workplace-based assessment, ERS is not in the position, authoritative and financially, to carry out these assessments.

It was suggested that the ERS might provide innovative approaches to the assessment of knowledge and skills through a gamification approach based, for instance, on the objective structured clinical examination model of test "stations" based on the eight ERS topic areas. These can be presented at the International Congress, on the website and on mobile devices. This will give members feedback on their performance and advice about education and training opportunities they might access as well as providing ERS with an increasing database of information about educational needs to inform future provision. In addition, ERS reflected on a system that records and recognises the everyday CPD process that clinicians undertake.

The patient experience in medical practitioner training

One of the cornerstones of medical practice is development of the physician-patient relationship within the consultation. Each consultation is modified by the prior expectations of the participants and illness-related worries held by the patient. SouthGate and Bass [21] describe how to record the worries and expectations of patients visiting their physician and changes occurring as a result of the consultation. The major worries of the group were about discomfort, effects of illness on patients and their family, the prospect of a physical examination, and about explaining the problem to the physician. Prominent expectations were for an explanation of diagnosis and treatment, and a friendly and understanding manner from the physician. These findings are relevant to respiratory medicine and should always be considered by the physician who must put the patient at ease.

We clarified early on in our discussion that we would not focus on "expert patients", who may have rare diseases, working to ensure resources are made available for research and improvements in clinical care. We considered what any patient should expect when consulting their doctor, especially if they are seen with students present. They become teachers at that point, and can be encouraged to speak, and ask and answer questions [22].

Next, we discussed the safeguards for patients in their role as teacher and agreed the responsibilities of clinicians who are supervising the encounter.

The ERS will continue to present the patient perspective in educational programmes to ensure they have a role to play in contributing to programmes that fit their needs and have a key role in providing feedback to physicians on their performance.

\section{Take-home message}

The ERS has a solid foundation of CPD activities and has developed systems to support these services. However, medical education is not static, and the ERS is continuing to reflect on and develop its strategic perspective, especially in terms of the effectiveness of the education and training provided for the individual physician and the service. The seminar touched on issues of curriculum design, learning methods and assessment, as well as the central topics of funding and patient involvement. While applauding the current provision, it was recognised that crucial elements now need to be included. These are the recognition and support for the everyday CPD that every clinician undertakes as part of their professional practice, and information about both the need for specific CPD provision and information about the effect of ERS activities on practice or on the individual practitioner.

Perhaps the greatest challenge for ERS Education is the design and implementation of an educational programme that meets individual needs of ERS membership whilst also aspiring to improve patient care. The ERS does not wish to engage in development of international recertification criteria, the vision is to create a framework to help members to identify learning needs and offer practicing specialists the opportunity to update their knowledge, skills and professional development to 
begin to meet the changing demands of "modern" medicine [23].

The following factors for success were identified.

- Raising awareness about the effectiveness of a robust CPD system

- Provide means for gap analysis helping practitioners to be aware that they "do not know what they do not know"

- Offer a flexible and appropriately assessed or reviewed approach to update knowledge, skills and behaviours on a self-dictated, repeatable, manageable and voluntary basis

The ERS is currently in the exploratory phase of this important initiative. The next steps include the identification of topics for trained specialists on eight respiratory diseases, and development of flexible, adaptable and optional programmes that may guide respiratory physicians on how to keep up to date in key areas.
The delivery of CPD with the end goal of improving practice performance and patient outcomes is a complex task, and will require constant attention from ERS. "The reality of the future of healthcare is the inevitable need to work without borders" [24]. Medical education experts still discuss, debate and challenge how best to strike a balance between offering some structure to trained physicians in revalidation while ensuring that this structure is flexible and adaptable to the needs of each individual lifelong learner. The success of this overarching aspiration is bound by the need for not only alignment across each education pillar: curriculum, programmes, e-learning and assessments but that these four pillars are seamlessly integrated in the delivery of activities. If the ERS can succeed in proposing respiratory specialists this flexible structure for their CPD learning needs, then we move one step closer to a process of training that improve quality of care for patients across Europe.

\section{Affiliations}

Sharon Mitchell ${ }^{1}$, Carolin Sehlbach², Erik Driessen², Amy Farr ${ }^{3}$, Janet Grant ${ }^{4}$, Lars Konge ${ }^{5}$, Carine Pannetier ${ }^{3}$, Suzanne Schut ${ }^{2}$, Daiana Stolz ${ }^{6}$, Gernot Rohde ${ }^{7}$

${ }^{1}$ World Heart Federation, Geneva, Switzerland. ${ }^{2}$ Dept of Educational Development and Research, Faculty of Health, Medicine and Life Sciences, Maastricht University, Maastricht, The Netherlands. ${ }^{3}$ European Respiratory Society, Lausanne, Switzerland. ${ }^{4}$ CenMEDIC, Hampton, UK. ${ }^{5}$ Copenhagen University and the Capital Region of Denmark, Centre for Clinical Education, University of Copenhagen, Copenhagen, Denmark. ${ }^{6}$ University Hospital Basel, Pulmonary Care Division, Basel, Switzerland. ${ }^{7}$ Respiratory Medicine, Johann Wolfgang Goethe University Hospital, Frankfurt, Germany.

\section{Conflict of interest}

S. Mitchell is a former employee of the European Respiratory Society. C. Sehlbach has nothing to disclose. E. Driessen has nothing to disclose. A. Farr is an employee of the European Respiratory Society. J. Grant has nothing to disclose. L. Konge has nothing to disclose. C. Pannetier is an employee of the European Respiratory Society. S. Schut has nothing to disclose. D. Stolz has nothing to disclose. G. Rohde reports personal fees from Pfizer, Boehringer Ingelheim, Solvay, GSK, Essex Pharma, MSD, Grifols, Chiesi, Vertex, Berlin Chemie, Astra-Zeneca, Bayer, Roche and Novartis, outside the submitted work.

\section{References}

1. Wojtczak A. Occasional Paper 03: Glossary of Medical Education Terms. Dundee, AMEE, 2003

2. Miller SH, Thompson JN, Mazmanian PE, et al. Continuing medical education, professional development, and requirements for medical licensure: a white paper of the Conjoint Committee on Continuing Medical Education. J Contin Educ Health Prof 2008; 28: 95-98.

3. Moore DE, Green JS, Gallis HA. Achieving desired results and improved outcomes: integrating planning and assessment throughout learning activities. J Contin Educ Health Prof 2009 29: 1-15.

4. Sehlbach C, Govaerts MJ, Mitchell S, et al. Box-ticking and Olympic high jumping -physicians' perceptions and acceptance of national physician validation systems. Med Teach 2018: 1-6.

5. Sehlbach C, Govaerts MJ, Mitchell S, et al. Doctors on the move: a European case study on the key characteristics of nationa recertification systems. BMJ Open 2018; 8: e019963.

6. Lilley P, Harden R. Standards and medical education. Med Teach 2003; 25: 349-351.

7. Boulet J, Zanten M. Ensuring high-quality patient care: the role of accreditation, licensure, specialty certification and revalidation in medicine. Med Educ 2014; 48: 75-86.
8. Sherbino J, FrankJR. Educational Design: A CanMEDS Guide for the Health Professional. Ottawa, Royal College of Physicians and Surgeons of Canada, 2011.

9. Miller GE. The assessment of clinical skills/competence/ performance. Acad Med 1990; 65: S63-S67.

10. Noël J-L, Séverin T, Bloch K. The European Diploma in Adult Respiratory Medicine: a label of quality for adult respiratory medical specialists. Breathe 2010; 6: 191-194.

11. Pitta F, Mitchell S, Chatwin M, et al. A core syllabus for post-graduate training in respiratory physiotherapy. Breathe 2014; 10: 220-228.

12. Artigas A, Vassilakopoulos T, Brochard L, et al. Respiratory Critical Care HERMES: a European core syllabus in respiratory critical care medicine. Breathe 2012; 8: 216-229.

13. Gamarra F, Noël J-L, Brunelli A, et al. Thoracic oncology HERMES: European curriculum recommendations for training in thoracic oncology. Breathe 2016; 12: 249-255.

14. Mitchell S, Riha RL, Rohde G, et al. Continuing professional development: introducing the ERS International Certificate in Respiratory Sleep Medicine. Breathe 2017 13: 11-14. 
15. Loddenkemper R, Séverin T, Eiselé J, et al. HERMES: a European core syllabus in respiratory medicine. Breathe 2006; 3: 59-69.

16. Farr A, Clementsen P, Herth F, et al. Endobronchial ultrasound: launch of an ERS structured training programme. Breathe 2016; 12: 217-220.

17. Aragon SR, Johnson ES. Factors influencing completion and noncompletion of community college online courses. Am J Distance Educ 2008; 22: 146-158.

18. Van Tartwijk J, Driessen EW. Portfolios for assessment and learning: AMEE Guide no. 45. Med Teach 2009; 31: 790-801.

19. Kromann $C B$, Jensen $M L$, Ringsted $C$. The effect of testing on skills learning. Med Educ 2009; 43: 21-27.
20. Konge L, Clementsen PF, Ringsted C, et al. Simulator training for endobronchial ultrasound: a randomised controlled trial. Eur Respir J 2015; 46: 1140-1149.

21. Southgate LJ, Bass MJ. Determination of worries and expectations of family practice patients. J Fam Pract 1983; 16: 339-344.

22. Stacy R, Spencer J. Patients as teachers: a qualitative study of patients' views on their role in a community-based undergraduate project. Med Educ 1999; 33: 688-694.

23. Mitchell S, Bloch KE, Butiene I, et al. "Education is the passport to the future": enabling today's medical teachers to prepare tomorrow's respiratory health practitioners. Eur Respir J 2014; 44: 578-584.

24. Wass V, Southgate L. Doctors without borders. Acad Med 2017; 92: 441-443. 